\title{
A RING WITH ARITHMETICAL CONGRUENCE LATTICE NOT PRESERVED BY ANY PIXLEY FUNCTION
}

\author{
IVAN KOREC
}

ABstract. A ring $(A ;+, \cdot)$ is constructed such that the congruence lattice $L_{A}$ of the ring $(A ;+, \cdot)$ is distributive, the elements of $L_{A}$ are pairwise permutable and there is no $L_{A}$-compatible function $p$ on $A$ such that

$$
p(a, b, b)=p(a, b, a)=p(b, b, a)=a \text { for all } a, b \in A \text {. }
$$

1. Introduction and notation. Let $\mathrm{Eq}(A)$ denote the lattice of all equivalence relations on a set $A$. A sublattice $L$ of $\operatorname{Eq}(A)$ is said to be arithmetical if $L$ is distributive and the elements of $L$ are pairwise permutable. For arbitrary $\mathfrak{D} \in$ $\mathrm{Eq}(A)$ and $\left(a_{1}, \ldots, a_{k}\right),\left(b_{1}, \ldots, b_{k}\right) \in A^{k}$ we write

$$
\left(a_{1}, \ldots, a_{k}\right) \mathfrak{D}\left(b_{1}, \ldots, b_{k}\right)
$$

if $a_{1} \mathfrak{D} b_{1} \wedge \cdots \wedge a_{k} \mathfrak{D} b_{k}$. A mapping $f$ of [a subset of] $A^{k}$ into $A$ is said to be a $k$-ary $\mathfrak{D}$-compatible [partial] function if (1.1) implies

$$
f\left(a_{1}, \ldots, a_{k}\right) \mathscr{D}\left(b_{1}, \ldots, b_{k}\right)
$$

for all $\left(a_{1}, \ldots, a_{k}\right),\left(b_{1}, \ldots, b_{k}\right) \in \operatorname{Dom}(f)$. For $L \subseteq \operatorname{Eq}(A), f$ is said to be $L$-compatible if it is $\mathscr{D}$-compatible for every $\mathfrak{D} \in L$. A ternary function $p$ on the set $A$ is said to be a Pixley function if

$$
p(a, b, b)=p(a, b, a)=p(b, b, a)=a \text { for all } a, b \in A .
$$

Let an arithmetical lattice $L \subseteq \operatorname{Eq}(A)$ be the congruence lattice of an algebra over $A$. It was proved in [9] that if $L$ is finite then there is an $L$-compatible Pixley function. At the Colloquium on Universal Algebra in Oberwolfach, July, 1973, A. F. Pixley asked whether the finiteness of $L$ can be omitted in the mentioned theorem. Partial positive answers are contained in [7] and [8]; e.g. the finiteness of $L$ can be replaced by the countability of $A$. In the present paper it is shown that the finiteness of $L$ cannot be completely omitted.

A related problem is solved in [3] where congruence equalities are defined; for example, arithmeticity corresponds to the congruence equality $(\xi \eta) \cap(\zeta \xi)=$ $\xi(\eta \cap \zeta)$. Further, local Mal'cev characterisability is introduced, and it is proved that arithmeticity is the only nontrivial congruence equality which is locally Mal'cev characterisable.

Received by the editors May 29, 1980; presented at the Summer School of Universal Algebra and Ordered Sets, September 1-10, 1980, Kroměriž, Czechoslovakia.

AMS (MOS) subject classifications (1970). Primary 08A25, Secondary 08A05. 
$L_{Z}$ denotes the congruence lattice of the ring $(Z,+, \cdot)$ of integers and $\omega$ the set of nonnegative integers. The symbol

$$
\left(\begin{array}{l}
n \\
i
\end{array}\right)=\frac{n(n-1) \cdots(n-i+1)}{i !}
$$

is defined for all $n \in Z$ and $i \in \omega$. The (nonnegative) least common multiple of integers $a_{1}, \ldots, a_{n}$ is denoted 1.c.m. $\left(a_{1}, \ldots, a_{n}\right)$. Square brackets are used for the integer part. Sequences of integers (i.e. mappings of $\omega$ into $Z$ ) are denoted $x, y, z$, $u, v, t \ldots$ The $n$th member of the sequence $x$ is denoted $x_{n}$; every sequence begins with the 0 th member. Hence $x=\left(x_{0}, x_{1}, x_{2}, \ldots\right)$, and analogously for other letters. The formula

$$
x \equiv y \quad(\bmod t)
$$

means $x_{n} \equiv y_{n}\left(\bmod \left|t_{n}\right|\right)$ for all $n \in \omega ; a \equiv b(\bmod 0)$ means $a=b$; and $a \equiv b$ (mod 1) holds for arbitrary integers $a, b$. We also generalize (1.5) for $k$-tuples of sequences similarly to (1.1).

2. Lower bound for $L_{Z}$-compatible Pixley functions. We shall need some results from [4], which are summarized in the next theorem.

2.1. THEOREM. Let $f$ be a mapping of $\omega$ into $Z$. Then $f$ can be uniquely represented in the form

$$
f(k)=\sum_{i=0}^{\infty} A_{i}\left(\begin{array}{c}
k \\
i
\end{array}\right) .
$$

The numbers $A_{i}$ are integers and for every $n \in \omega$

$$
A_{n}=\sum_{i=0}^{n}(-1)^{i}\left(\begin{array}{c}
n \\
i
\end{array}\right) f(n-i) .
$$

Further, $f$ is $L_{Z}$-compatible if and only if for every $n \in \omega$ the number $A_{n}$ is a multiple of 1.c.m. $(1,2, \ldots, n)$.

The formula (2.1.2) can be proved from (2.1.1) and also by [6, formula 34, p. 438].

The paper [4] also contains a lower bound for so-called genuine pseudopolynomials. There are several reasons why the bound from [4] cannot be immediately used here; the most substantial one is that the bound in [4] is not uniform. We shall find a uniform exponential lower bound for $L_{Z}$-compatible Pixley functions; however, we shall not look for the best numerical result. To formulate our result briefly we define

2.2. Definition. Let $M_{0}=0, M_{1}=1$ and for all $n \in \omega, n>2$ let

$$
M_{n}=\left[\operatorname{l.c.m} .(1,2, \ldots, k) / 2^{k}\right]
$$

where $k$ is the greatest prime not greater than $n$.

By [4] or [1] the sequence $\left(M_{0}, M_{1}, M_{2}, \ldots\right)$ increases exponentially. More precisely,

$$
\lim _{n \rightarrow \infty} \log \left(2^{n} \cdot M_{n}\right) / \log \left(e^{n}\right)=1
$$

where $e=2.71828 \ldots$ 
2.3. TheOREM. For every $L_{\boldsymbol{Z}}$-compatible Pixley function $p$ and every $n \in \boldsymbol{\omega}$

$$
\max \{|p(a, b, c)| ; a, b, c \in\{-n,-n+1, \ldots, n-1, n\}\}>M_{n} .
$$

Proof. For $n \leqslant 1$ we have $|p(n, n, n)|=|n|=n=M_{n}$, hence (2.3.1) holds. Now assume $n>1$; without loss of generality we may assume that $n$ is a prime. Denote

$$
f(k)=p(k, k-n, 0) .
$$

It obviously suffices to show

$$
\max \{|f(k)| ; k \in\{0,1, \ldots, n\}\}>M_{n} .
$$

The partial function $f$ is $L_{Z}$-compatible and hence for every $k \in \omega$ we have $f(k)=p(k, k-n, 0) \equiv p(k, k, 0)=0(\bmod n)$. Further, $f(0)=p(0,-n, 0)=0$, $f(n)=p(n, 0,0)=n$. There are integers $A_{i}$ such that (2.1.1) holds for all $k \in \omega$; then (2.1.2) holds, too. For every $i \in\{1,2, \ldots, n-1\}$ the prime $n$ divides both $\left(\begin{array}{l}n \\ i\end{array}\right), f(n-i)$ and hence

$$
\left(\begin{array}{c}
n \\
i
\end{array}\right) f(n-i) \equiv 0 \quad\left(\bmod n^{2}\right)
$$

Therefore

$$
A_{n} \equiv f(n)+(-1)^{n} f(0)=n \quad\left(\bmod n^{2}\right) .
$$

Hence $A_{n} \neq 0$. On the other hand, (2.1.2) implies

$$
\left|A_{n}\right| \leqslant \sum_{i=0}^{n}\left(\begin{array}{c}
n \\
i
\end{array}\right) f(n-i)<2^{n} \max \{|f(k)| ; k \in\{0,1, \ldots, n\}\} .
$$

If (2.3.3) does not hold then we have

$$
0 \neq\left|A_{n}\right|<\left|2^{n} \cdot M_{n}\right|<\text { l.c.m. }(1,2, \ldots, n) .
$$

Therefore $A_{n}$ is not a multiple of 1.c.m. $(1,2, \ldots, k)$ which contradicts the $L_{z}$-compatibility of $f$.

3. The example. A sequence of integers $\left(x_{0}, x_{1}, x_{2}, \ldots\right)$ is said to be polynomially bounded if there is $k \in \omega$ such that $\left|x_{n}\right|<(n+2)^{k}$ for all $n \in \omega$. The memberwise product and the memberwise difference of two polynomially bounded sequences of integers are polynomially bounded. Therefore the set of polynomially bounded sequences forms a ring with these operations. This ring will be called the ring of polynomially bounded sequences.

3.1. ThEOREM. Let $L_{A}$ be the congruence lattice of the ring $(A ;+, \cdot)$ of polynomially bounded sequences. Then $L_{A}$ is arithmetical and there is no $L_{A}$-compatible Pixley function.

The proof will be divided into several lemmas. Throughout the whole present section $A$ and $L_{A}$ will always have the meaning from Theorem 3.1.

3.2. LeMMA. $L_{A}$ is arithmetical.

Proof. The elements of $L_{A}$ are obviously pairwise permutable; it remains to prove that $L_{A}$ is distributive. 
Let $\xi, \eta, \zeta \in L_{A}, x, y, z \in A$ and $x \xi y, x \eta z \zeta y$. We have to find $u \in A$ such that

$$
x(\xi \cap \eta) u(\xi \cap \zeta) y .
$$

For every $n \in \omega$ consider the system of congruences

$$
\begin{array}{ll}
u_{n} \equiv x_{n} & \left(\bmod \left|x_{n}-y_{n}\right|\right), \\
u_{n} \equiv x_{n} & \left(\bmod \left|x_{n}-z_{n}\right|\right), \\
u_{n} \equiv y_{n} & \left(\bmod \left|y_{n}-z_{n}\right|\right),
\end{array}
$$

with the unknown $u_{n}$. Every pair of congruences (3.2.2) is solvable. Hence by the Chinese remainder theorem the system (3.2.2) is solvable. Moreover, if the integers $x_{n}, y_{n}, z_{n}$ are pairwise different we can arrange

$$
\left|u_{n}\right|<\left|\left(x_{n}-y_{n}\right) \cdot\left(x_{n}-z_{n}\right) \cdot\left(y_{n}-z_{n}\right)\right| ;
$$

otherwise $u_{n}$ will be the repeated integer from $x_{n}, y_{n}, z_{n}$. Then the sequence $u=\left(u_{0}, u_{1} u_{2}, \ldots\right)$ is polynomially bounded and hence $u \in A$. Since (3.2.2) holds for all $n \in \omega$ we have $u \xi x, u \eta x, u \xi y$ which together with $x \xi y$ implies (3.2.1). Q.E.D.

The next lemma obviously holds for any $k$-ary $L_{A}$-compatible function; since we need it only for $k=3$ we formulate it only for this case to avoid more complicated notation.

3.3. LEMMA. For every ternary $L_{A}$-compatible function $p$ there is a unique sequence $\left(p_{0}, p_{1}, p_{2}, \ldots\right)$ of ternary $L_{Z}$-compatible functions such that

$$
\begin{aligned}
p\left(\left(x_{0}, x_{1}, x_{2}, \ldots\right),\left(y_{0}, y_{1}, y_{2}, \ldots\right),\left(z_{0}, z_{1}, z_{2}, \ldots\right)\right) \\
\quad=\left(p_{0}\left(x_{0}, y_{0}, z_{0}\right), p_{1}\left(x_{1}, y_{1}, z_{1}\right), p_{2}\left(x_{2}, y_{2}, z_{2}\right), \ldots\right)
\end{aligned}
$$

for all $\left(x_{0}, x_{1}, x_{2}, \ldots\right),\left(y_{0}, y_{1}, y_{2}, \ldots\right),\left(z_{0}, z_{1}, z_{2}, \ldots\right) \in A$.

Proof. For every $n \in \omega$ denote by $\mathfrak{D}_{n}$ the least element of $L_{A}$ such that

$$
(1, \ldots, 1,0,1,1, \ldots) \mathscr{D}_{n}(0,0,0, \ldots)
$$

where 0 on the left-hand side is at the $n$th place. Since the function $p$ is $\mathfrak{D}_{n}$-compatible the $n$th member of $p(x, y, z)$ depends only on the $n$th members $x_{n}, y_{n}, z_{n}$ of $x, y, z$, respectively. Therefore there are ternary functions $p_{0}, p_{1}, p_{2}, \ldots$ such that (3.3.1) holds; the uniqueness is obvious. It remains to prove that the functions $p_{0}, p_{1}, p_{2}, \ldots$ are $L_{Z}$-compatible. Let $a_{1}, a_{2}, a_{3}, b_{1}, b_{2}, b_{3} \in Z, n, d \in \omega$ and $\left(a_{1}, a_{2}, a_{3}\right) \equiv\left(b_{1}, b_{2}, b_{3}\right)(\bmod d)$. Let the $n$th members of the sequences $x, y$, $z, u, v, w, t$ be $a_{1}, a_{2}, a_{3}, b_{1}, b_{2}, b_{3}, d$, respectively, and let all the remaining members of these sequences be zero. Then

$$
(x, y, z) \equiv(u, v, w) \quad(\bmod t)
$$

and since $p$ is $L_{A}$-compatible

$$
p(x, y, z) \equiv p(u, v, w) \quad(\bmod t) .
$$

Therefore by (3.3.1)

$$
p_{n}\left(x_{n}, y_{n}, z_{n}\right) \equiv p_{n}\left(u_{n}, v_{n}, w_{n}\right) \quad\left(\bmod t_{n}\right)
$$


i.e.

$$
p_{n}\left(a_{1}, a_{2}, a_{3}\right) \equiv p_{n}\left(b_{1}, b_{2}, b_{3}\right) \quad(\bmod d)
$$

Q.E.D.

3.4. Lemma. There is no $L_{A}$-compatible Pixley function.

Proof. Let $p$ be such a function. By Lemma 3.3 there are $L_{z}$-compatible functions $p_{0}, p_{1}, p_{2}, \ldots$ such that (3.3.1) holds; $p_{0}, p_{1}, p_{2}, \ldots$ are obviously Pixley functions. Hence for every $n \in \omega$ there are integers $x_{n}, y_{n}, z_{n} \in\{-n,-n+$ $1, \ldots, n-1, n\}$ such that

$$
\left|p_{n}\left(x_{n}, y_{n}, z_{n}\right)\right|>M_{n} \text {. }
$$

The sequences $x=\left(x_{0}, x_{1}, x_{2}, \ldots\right), y=\left(y_{0}, y_{1}, y_{2}, \ldots\right), z=\left(z_{0}, z_{1}, z_{2}, \ldots\right)$ belong to $A$, hence $t=\left(t_{0}, t_{1}, t_{2}, \ldots\right)=p(x, y, z)$ also belongs to $A$. However, for every $n \in \omega,\left|t_{n}\right|=\left|p_{n}\left(x_{n}, y_{n}, z_{n}\right)\right| \geqslant M_{n}$. Hence $t$ is not polynomially bounded, which contradicts $t \in A$.

\section{REFERENCES}

1. K. Chandrasekharan, Arithmetical functions, Russian transl., "Nauka", Moscow, 1975.

2. G. Grätzer, Universal algebra, Van Nostrand, Princeton, N.J., 1968.

3. H. P. Gumm, Is there a Mal'cev theory for single algebras?, Algebra Universalis 8 (1978), 320-329.

4. R. R. Hall, On pseudopolynomials, Mathematika 18 (1971), 71-77.

5. B. Jonsson, Topics in universal algebra, Lecture Notes in Math., vol. 250, Springer-Verlag, Berlin and New York, 1970.

6. J. Kaucký, Combinatorial identities, Veda, Bratislava, 1975.

7. I. Korec, A ternary function for distributivity and permutability of an equivalence lattice, Proc. Amer. Math. Soc. 69 (1978), 8-10.

8. Concrete representation of some equivalence lattices, Math. Slovaca (to appear).

9. A. F. Pixley, Completeness in arithmetical algebras, Algebra Universalis 2 (1972), 179-196.

10. _ Distributivity and permutability of congruence relations in equational classes of algebras, Proc. Amer. Math. Soc. 14 (1963), 105-109.

11. __ Local Mal' cev conditions, Canad. Math. Bull. 15 (1972), 559-568.

Department of Algebra, Faculty of Mathematics and Physics, University of Comenius, 81631 Bratislava, Czechoslovakia 